\title{
Doble primario sincrónico: condrosarcoma y sarcoma fusocelular de alto grado asociado a síndrome de Maffucci
}

\author{
Synchronous primary double: condrosarcoma and sarcoma high \\ grade fusocelular associated with Maffucci syndrome
}

\author{
García-Ortega DY,* Clara-Altamirano MA, ${ }^{\ddagger}$ Álvarez-Cano A, ${ }^{\S}$ Partida-Nava GV, \\ Martínez-Said H,* Caro-Sánchez CHS," Cuellar-Hubbe M**
}

Instituto Nacional de Cancerología SS.

RESUMEN. El síndrome de Maffucci se caracteriza por la presencia de múltiples encondromas y hemangiomas que pueden afectar tejidos blandos y otros órganos. El riesgo de transformación maligna de las lesiones es de 100\% durante la vida del individuo, siendo el condrosarcoma el tumor maligno más frecuentemente asociado. Se presenta el caso de un hombre de 44 años de edad con diagnóstico de síndrome de Maffucci, el cual desarrolló un doble primario sincrónico: condrosarcoma y sarcoma fusocelular de alto grado multicéntrico de región escapular y tricipital, fue tratado con desarticulación interescapulotorácica, mostró progresión acelerada y enfermedad pulmonar. Existen otras neoplasias asociadas al síndrome de Maffucci tales como adenocarcinoma de páncreas, tumores mesenquimales de ovario, gliomas, astrocitomas y tumores de la pituitaria. Por lo que resulta muy interesante reportar la asociación infrecuente entre un sarcoma fusocelular y un condrosarcoma secundario en pacientes con síndrome de Maffucci. El seguimiento en este grupo de pacientes es complejo y se basa en la búsqueda intencionada de lesiones con crecimiento acelerado, prestando atención en lesiones de crecimiento progresivo, síntomas clínicos o datos radiológicos de malignidad.

Palabras clave: Encondroma, hemangioma, dolor, tratamiento.
ABSTRACT. Maffucci syndrome is characterized by the presence of multiple enchondromes and hemangiomas that can affect soft tissues and other organs. The risk of malignant transformation of lesions is $100 \%$ during the life of the individual, with chondrosarcoma being the most frequently associated malignant tumor. We present the case of a 44-year-old man diagnosed with Maffucci syndrome who developed a synchronous double primary: chondrosarcoma and high-grade multicenter fusocellular sarcoma of scapular and tricipital region, was treated with disarticulation interscapule-thoracic, presented accelerated progression and lung disease. There are other neoplasms associated with Maffucci syndrome, such as pancreatic adenocarcinoma, mesenchymal ovarian tumors, gliomas, astrocytomas and pituitary tumors. It is therefore very interesting to report the uncommon association between fusocellular sarcoma and secondary chondrosarcoma in patients with Maffucci syndrome. Follow-up in this group of patients is complex and is based on the intentional search for accelerated growing lesions, paying attention to progressive growth injuries, clinical symptoms or radiological malignancy data.

Keywords: Enchondroma, hemangioma, pain, treatment.

\footnotetext{
* Cirujano Oncólogo, adscrito al Departamento de Piel y Partes Blandas del Instituto Nacional de Cancerología (CDMX).

‡ Ortopedista Oncólogo, adscrito al Departamento de Piel y Partes Blandas del Instituto Nacional de Cancerología (CDMX).

${ }^{\S}$ Cirujano Oncólogo, adscrito al Hospital Universitario, UANL.

" Residente de cirugía oncológica, Instituto Nacional de Cancerología (CDMX).

" Patólogo Oncólogo, adscrito al Departamento de Piel y Partes Blandas del Instituto Nacional de Cancerología (CDMX).

** Cirujano Oncólogo, jefe del Departamento de Piel y Partes Blandas del Instituto Nacional de Cancerología (CDMX).
}

Dirección para correspondencia:

Clara-Altamirano MA

Avenida San Fernando Núm. 22, Col. Sección XVI, C.P. 14080, Alcaldía Tlalpan, Ciudad de México.

Teléfono: 56280400 y 56554766

E-mail: drmiguelclara@gmail.com

Este artículo puede ser consultado en versión completa en: www.medigraphic.com/actaortopedica 


\section{Introducción}

El síndrome de Maffucci fue descrito por Angelo Maffucci en 1881, es una enfermedad extraordinariamente rara, con menos de 200 casos reportados en la literatura. No se cuenta con estadísticas sobre su prevalencia. Se presenta como encondromatosis múltiple asociada a hemangiomas de tejidos blandos que pueden afectar piel, tejido subcutáneo u otros órganos. ${ }^{1}$

El síndrome de Maffucci se caracteriza por la presencia de múltiples encondromas y hemangiomas que pueden afectar tejidos blandos y otros órganos. ${ }^{2}$ Está relacionado con mutaciones somáticas en el gen que codifica la isocitrato deshidrogenasa 1 y 2 (IDH1, IDH2). IDH1 participa en el metabolismo de la glucosa y de los ácidos grasos. El mecanismo por el cual se produce la formación de encondromas no está bien definido. El riesgo de transformación maligna de las lesiones es de $100 \%$ durante la vida del individuo, puede ser en forma de condrosarcoma, angiosarcoma o linfangiosarcoma. No se ha documentado la presentación sincrónica de dos sarcomas en estos pacientes. ${ }^{3}$

Las encondromatosis múltiples pueden manifestarse de forma esporádica (sin asociación a un patrón hereditario) o de forma hereditaria; habitualmente son lesiones benignas, aunque poseen potencial de transformación maligna. Los encondromas pueden encontrarse en cualquier sitio, están descritos incluso en la tráquea, es más frecuente observarlos en las metáfisis de huesos largos. ${ }^{4}$ La distribución suele ser asimétrica y ocasionan crecimiento dispar de las extremidades, además tienen variabilidad en tamaño, número, localización, edad y evolución. Los problemas clínicos asociados son deformidades en el esqueleto, dolor, limitación funcional de las extremidades y riesgo potencial de malignidad.

Se presenta el caso de un hombre de 44 años de edad con diagnóstico de síndrome de Maffucci, el cual desarrolló un doble primario sincrónico: condrosarcoma y sarcoma fusocelular de alto grado multicéntrico de región escapular y tricipital, fue tratado con desarticulación interescapulotorácica, mostró progresión acelerada y enfermedad pulmonar. Se expone el caso y se hace revisión de la literatura.

\section{Reporte de caso}

Hombre de 44 años, con talla baja asociado a asimetría de extremidades y presencia de múltiples nódulos subcutáneos. Siete meses previos a su ingreso a nuestro centro evidencia aumento de volumen de región escapular derecha, con parestesias, disminución de fuerza y dolor, clínicamente presenta acortamiento de la extremidad superior derecha e inferior izquierda, además muestra lesión de $20 \mathrm{~cm}$ en región escapular derecha, pétrea, fija con una zona de ulceración en la región deltoidea, hay limitación de la fuerza y flexoextensión de la articulación glenohumeral ipsilateral así como hipotrofia tenar e hipotenar, asociada a múltiples nódulos subcutáneos de 1 a $3 \mathrm{~cm}$, el mayor en la región tricipital (Figura 1).

Como elementos del protocolo de estudio se realiza resonancia magnética nuclear (RMN) de hombro que muestra lesión de $18 \mathrm{~cm}$ de diámetro mayor, predominantemente hipointensa en T1 e hiperintensa en T2, con formación de trabéculas en su interior, sugestivo de formación de matriz condroide. La tomografía de tórax muestra múltiples encondromas en cinco arcos costales, sin enfermedad pulmonar; la serie ósea presenta acortamiento humeral con una lesión de $15 \mathrm{~cm}$ productora de matriz condroide, múltiples encondromas de 0.5 a $1.5 \mathrm{~cm}$ los mayores, distribuidos en toda la extremidad, además de deformidad en metacarpo y falanges; se observan lesiones compatibles con encondromas en fémur distal izquierdo, costillas, húmero y radio izquierdo.

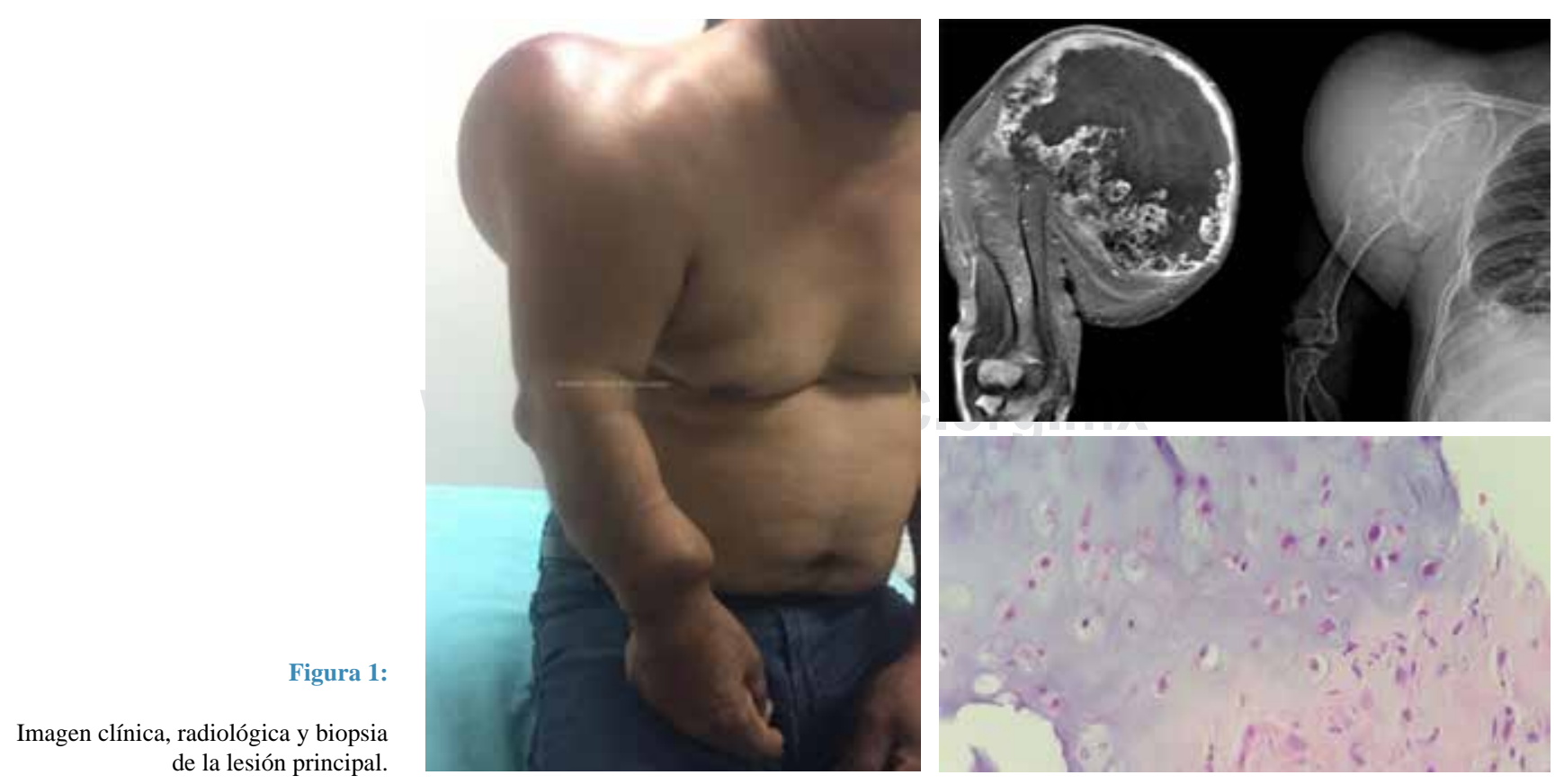


A nivel de cráneo sin lesiones sospechosas se realizó biopsia percutánea con aguja que reportó condrosarcoma convencional grado 2 (Figura 1).

Clínicamente, se integró diagnóstico de síndrome de Maffucci. Además de la lesión escapular se encontró uno de los nódulos subcutáneos con imagen sospechosa de transformación maligna. Debido al déficit neurológico presente en la extremidad, no se consideró al paciente candidato a preservación de la misma, ya que la expectativa de preservar una extremidad funcional no era razonable. Se realizó una desarticulación interescapulotorácica, se detectó lesión de $20 \mathrm{~cm}$ ulcerada, con deformidad y asimetría de húmero, múltiples encondromas, deformidad a nivel de epífisis y metáfisis distal de radio, así como metacarpo y falanges con acortamiento y deformación por la presencia de lesiones lobuladas de aspecto cartilaginoso. El reporte histopatológico fue un condrosarcoma grado 2, de $15 \mathrm{~cm}$ diámetro mayor, asociado a encondromas múltiples en húmero, cúbito, radio, metacarpo y falanges, además de múltiples hemangiomas en tejidos blandos. Se encontró segunda lesión en tríceps, separada anatómicamente de la lesión principal que fue diagnosticada como sarcoma fusocelular de alto grado no clasificable, de $4 \mathrm{~cm}$ diámetro mayor; sugestiva de un segundo primario. No se identificó invasión linfovascular o perineural y los márgenes quirúrgicos fueron negativos (Figura 2). El paciente presentó progresión acelerada y enfermedad pulmonar y falleció 13 meses después de la desarticulación.

\section{Discusión}

En el caso aquí expuesto se detectó una lesión anatómicamente separada de la lesión principal reportada como un sarcoma fusocelular con diferenciación vascular, lo que hace aún más raro el caso y complica las opciones terapéuticas que al paciente se le pudieran brindar.

En $81 \%$ de los pacientes con enfermedad de Ollier y 77\% con síndrome de Maffucci tienen mutaciones en IDH1 (98\%) e IDH2 (2\%). En los casos que no presentan esta mutación, se han descrito alteraciones en los genes que codifican el receptor de hormona paratiroidea (PTH1R), ${ }^{7,8,9,10} 10 \%$ de estos pacientes muestran una disminución de $30 \%$ en la función de este receptor. Sin embargo, el diagnóstico principal de estas patologías es clínico, apoyándonos en los reportes de patología.

Las complicaciones asociadas a estos síndromes incluyen fracturas espontáneas en $26 \%$, degeneración sarcomatosa, encondromas a condrosarcoma entre 15 y $40 \%$. Siendo el condrosarcoma la neoplasia más asociada al síndrome de Maffucci, ${ }^{8}$ otras neoplasias asociadas son angiosarcomas ${ }^{11} \mathrm{y}$ linfangiosarcomas. ${ }^{10}$

Los síndromes de Ollier y Maffucci, si bien son los más conocidos, no son los únicos. Los síndromes relacionados con el desarrollo de lesiones óseas que predisponen a transformación maligna pueden dividirse en hereditarios y esporádicos. La Tabla 1 muestra algunos de los síndromes asociados a lesiones óseas que aumentan el riesgo de desarrollar tumores malignos primarios del hueso (osteosarcoma y condrosarcoma).

En la serie más grande reportada a la fecha donde se analizan 144 pacientes con Ollier y 17 pacientes con Maffucci se encontró que $41 \%$ de los pacientes desarrolló uno o más condrosarcomas secundarios. La edad media de aparición fue de 30 años para pacientes con síndrome de Maffucci (entre 14-51 años). De esta población, 33\% tenían lesiones sincrónicas y 56\% metacrónicas. ${ }^{12}$
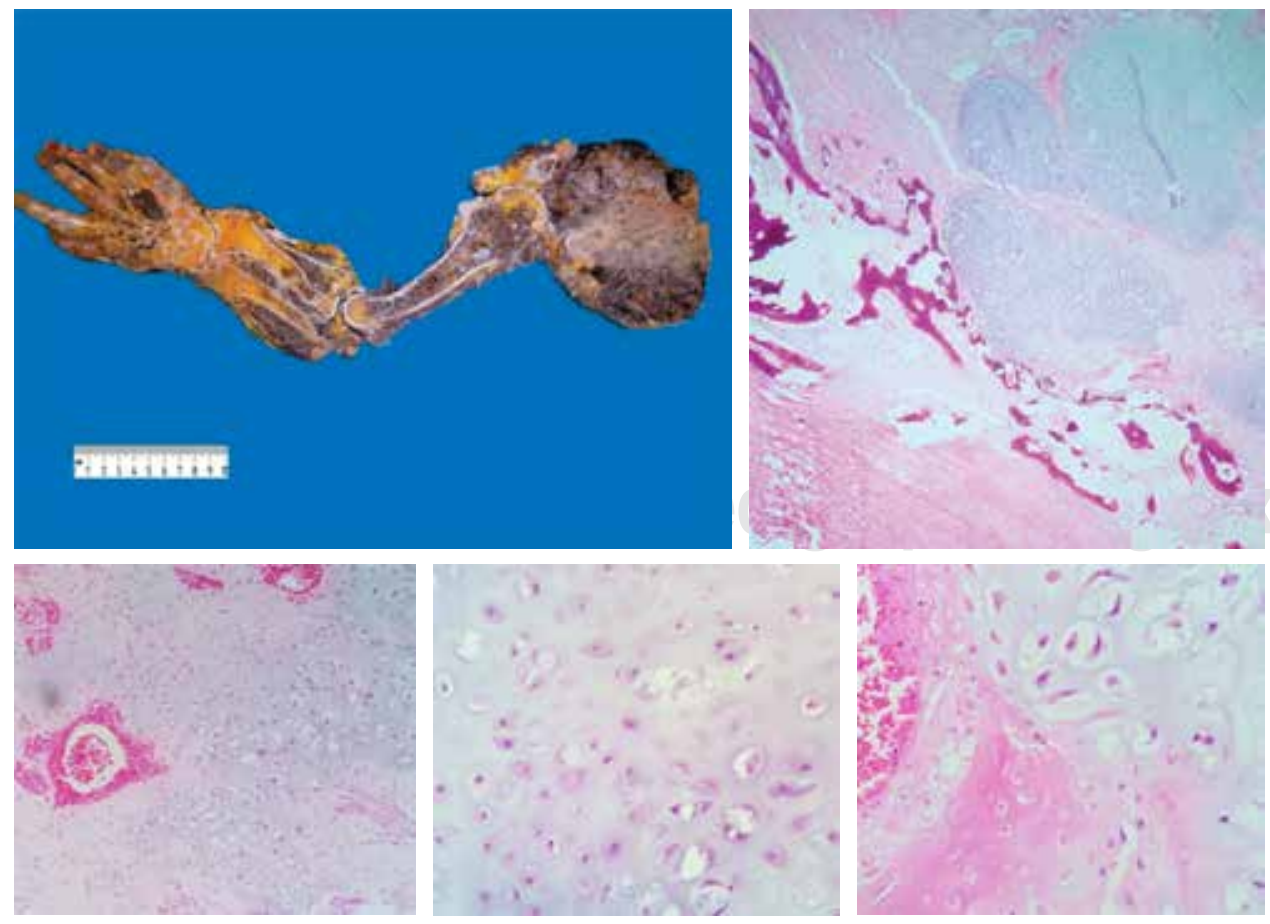

Figura 2:

Imagen de pieza quirúrgica y microscopía. Se observa tumor en sitio anatómico de escápula. Múltiples hemangiomas en deltoides, antebrazo y muñeca. 
Tabla 1: Síndromes asociados a anormalidades óseas y predisposición a neoplasias malignas.

\begin{tabular}{|c|c|c|c|}
\hline & $\begin{array}{l}\text { Anormalidad } \\
\text { genética }\end{array}$ & Características clínicas & Predisposición a \\
\hline \multicolumn{4}{|l|}{ Hereditarios } \\
\hline Exostosis múltiple familiar & EXT1 (8q24) & Formación de osteocondromas durante la infancia & Condrosarcoma \\
\hline & ЕXT2 (11p12) & Asimetría de extremidades & (0.5-5\% de los casos) \\
\hline Metacondromatosis & PTPN11 & Osteocondromas y encondromas múltiples & Condrosarcoma \\
\hline Sd. Rothmund-Thompson & $\begin{array}{l}\text { RecQL4 } \\
(8 q 24.3)\end{array}$ & Dermatosis, cataratas, aplasia radial, osteopenia & Osteosarcoma \\
\hline Sd. Rapandinilo & RecQL4 & $\begin{array}{l}\text { Hipoplasia radial, hipoplasia patelar, paladar hendido, dia- } \\
\text { rrea y luxaciones diversas, malformación de extremidades, } \\
\text { la inteligencia es normal y no hay anormalidades faciales }\end{array}$ & Osteosarcoma \\
\hline \multicolumn{4}{|l|}{ Esporádicos } \\
\hline Enf. Ollier & IDH1, IDH2 & Encondromas múltiples, asimetría de extremidades & Condrosarcoma \\
\hline Enf. Maffucci & IDH1, IDH2 & $\begin{array}{l}\text { Encondromas múltiples, asimetría de extremidades, } \\
\text { hemangiomas cutáneos o viscerales }\end{array}$ & Condrosarcoma \\
\hline
\end{tabular}

Existen otras neoplasias asociadas al síndrome de Maffucci, tales como adenocarcinoma de páncreas, tumores mesenquimales de ovario, gliomas, astrocitomas y tumores de la pituitaria. ${ }^{13}$ Por lo que resulta muy importante reportar esta asociación entre un sarcoma fusocelular y un condrosarcoma secundario en pacientes con síndrome de Maffucci. El seguimiento en este grupo de pacientes es complejo y se basa en la búsqueda intencionada de lesiones con crecimiento acelerado por tiempo prolongado, prestando atención en lesiones de crecimiento progresivo, síntomas clínicos o datos radiológicos de malignidad.

Dos sarcomas primarios pueden manifestarse de forma sincrónica o metacrónica. La biopsia de ambas lesiones, en caso de demostrar una histología diferente resuelve la duda sobre el tipo de presentación o si se trata de una recurrencia. No obstante, en caso de que una de las lesiones sea un sarcoma desdiferenciado, esta distinción es más compleja; el escenario más sencillo es que se demuestre la presencia de un componente de bajo grado en el sarcoma desdiferenciado. La inmunohistoquímica es también útil.

Las mutaciones de genes supresores tumorales como p53, INK4A o RB se encuentran en ambos subtipos de tumores y están relacionadas con la progresión tumoral. Es importante hacer notar que, sin embargo, no tienen interés en el diagnóstico. ${ }^{14,15,16,17} \mathrm{El}$ síndrome de Maffucci es una enfermedad poco frecuente, asociada a mutación somática y a un alto porcentaje de transformación maligna, siendo el condrosarcoma el tumor maligno más frecuente. La presencia de un doble primario sincrónico en el síndrome de Maffucci no se ha reportado previamente.

Bibliografía

1. Ngai C, Ding DY, Rapp TB. Maffucci syndrome. An interesting case and a review of the literature. Bulletin of the Hospital for Joint Diseases. 2015; 73(4): 282-5.

2. Maffucci AM. Di un caso di encondroma ed angioma multiplo. Contribuzione alla genesi embrionale dei tumori. Mov Med Chir. 1881; 3: 399-412.
3. Pansuriya TC, van Eijk R, d'Adamo P, et al. Somatic mosaic IDH1 and IDH2 mutations are associated with enchondroma and spindle cell hemangioma in Ollier disease and Maffucci syndrome. Nat Genet. 2012; 43(12): 1256-61.

4. Mahul B. FCAP. AJCC Cancer Staging Manual. $8^{\text {th }}$ ed., Springer, 2017.

5. Patrick P. The MD Anderson Cancer Care Series: Bone Sarcoma. New York: Springer Science + Business Media, 2013.

6. Goldblum J. Surgical pathology. $11^{\text {th }}$ ed. Chapter 40, Bone and Joints. Elsevier, 2017, 1740-1809.

7. Silve C. Ollier disease. Orphanet J Rare Dis. 2006; 1: 37.

8. Schwartz HS, Zimmerman NB, Simon MA, Wroble RR, Millar EA, Bonfiglio M. The malignant potential of enchondromatosis. $J$ Bone Joint Surg Am. 1987; 69(2): 269-74.

9. Zwenneke Flach H, Ginai AZ, Wolter Oosterhuis J. Best cases from AFIP. Maffucci syndrome: radiologic and pathologic findings. Radiographics. 2001; 21(5): 1311-6.

10. Auyeung J. Maffucci lymphangioma syndrome: an unusual variant of Ollier's disease, a case report and a review of the literature. $J$ Pediatr Orthop B. 2003; 12(2): 147-50.

11. Davidson TI, Kissin MW, Bradish CF, Westbury G. Angiosarcoma arising in a patient with Maffucci syndrome. Eur J Surg Oncol. 1985; 11(4): 381-4.

12. Verdegaal SH, Bovée JV, Pansuriya TC, Grimer RJ, Ozger H, Jutte PC, et al. Incidence, predictive factors, and prognosis of chondrosarcoma in patients with Ollier disease and Maffucci syndrome: an international multicenter study of 161 patients. Oncologist. 2011; 16(12): 1771-9.

13. Fernebro J, Carneiro A, Rydholm A, Domanski HA, Karlsson A, Borg A, et al. Genetic profiling differentiates second primary tumors from metastases in adult metachronous soft tissue sarcoma. Sarcoma. 2008; 2008: 431019. doi: 10.1155/2008/431019.

14. Chen S, Fritchie K, Wei S, Ali N, Curless K, Shen T, et al. Diagnostic Utility of IDH1/2 mutations to distinguish dedifferentiated chondrosarcoma from undifferentiated pleomorphic sarcoma of bone. Human Pathol. 2017; 65: 239-46.

15. Kunihiko Moriya, Mika K Kaneko, Xing Liu, Masami Hosaka, Fumiyoshi Fujishima, Jun Sakuma, et al. IDH2 and TP53 mutations are correlated with gliomagenesis in a patient with Maffucci syndrome. Cancer Sci. 2014; 105(3): 359-62.

16. Borden EC, Baker LH, Bell RS, Bramwell V, Demetri GD, Eisenberg BL, et al. Soft tissue sarcomas of adults: state of the translational science. Clin Cancer Res. 2003; 9(6): 1941-56.

17. Subramanian S, West RB, Marinelli RJ, Nielsen TO, Rubin BP, Goldblum JR, et al. The gene expression profile of extraskeletal myxoid chondrosarcoma. J Pathol. 2005; 206 (4): 433-44. 